Supporting Information for

\title{
Fragment Exchange Potential for Realizing Pauli Deformation of Inter-Fragment Interactions
}

Xin Chen, ${ }^{* 1,2}$ and Jiali Gao*2,3

1. Institute for Theoretical Chemistry, Jilin University, Changchun, Jilin, China

2. Shenzhen Bay Laboratory, Shenzhen, Guangdong, China

3. Department of Chemistry and Supercomputing Institute, University of Minnesota, MN 55455, USA

\section{Corresponding Author}

*Xin Chen: chenxin1211@mail.jlu.edu.cn, and Jiali Gao: gao@jialigao.org

This Supporting Information contains additional computational details, grid-level definition, optimization of the Yukawa potential, selection of Coulomb damping functions, effect of exchange polarization on electron density, and structures that show errors greater $3 \mathrm{kcal} / \mathrm{mol}$ in computed exchange energy (11 pages). 


\section{Additional computational details.}

In fragment-based electronic structure methods for large systems, the lack of explicit short-range exchange interactions between monomers can cause instability in self-consistent-field (SCF) optimization of the fragment molecular orbitals and can result in unphysical deformation in charge density, especially when large basis sets and diffuse functions are used. This problem is further exacerbated in molecular dynamics simulations in which deformed geometries and close contacts are often encountered. Although many-body expansion (MBE) approaches can provide two-body and many-body corrections to the energy, SCF convergence of mutually polarized fragmental wave functions remains a major challenge for macromolecular systems such as a solvated protein. from a formal requirement of $O\left(B^{4}\right)$ to $O\left(N_{\mathrm{f}} F^{4}\right)$, where $B$ is the number of basis functions in the entire system, $F$ is the average number of basis functions per fragment, and $N_{\mathrm{f}}$ is the number of fragments $\left(B=N_{\mathrm{f}} F\right)$. In this case, the computational cost increases linearly $\left(\operatorname{or} N_{\mathrm{f}} \log N_{\mathrm{f}}\right)$ with $N_{\mathrm{f}}$, in which the cost for one monomer is simply a prefactor, making such a fragment-based method particularly suitable for parallel computing

The Pauli deformation due to the XFP is obtained by an iterative procedure, in which the Kohn-Sham orbitals of each fragment is optimized under the combined effects of Hartree and exchange potential interactions (eq 6), until the changes in electron density and total energy converge to specific criteria. The overall process can be described by a double self-consistent field (DSCF) 
algorithm, featuring an inner, micro-SCF optimization of the fragmental orbitals and an outer, system-level SCF to determine the total energy. Intermediate matrices, numerical grids and electron integrals can be kept in memory throughout the DSCF process. Therefore, the extra computational cost is limited to the additional outer SCF iteration. In practice, an integrated variational procedure can be constructed, and the computation is performed as a single macro-SCF. For comparison, in the effective fragment potential (EFP) method, an inter-fragment Pauli repulsion potential was used with fixed parameters. ${ }^{4}$ In that case, the mutual exchange polarization effect is not explicit treated.

All calculations in this work were performed using a modified version of the PySCF software package. ${ }^{1}$ Benchmark BLW-ED analysis calculations were performed using a locally modified version of GAMESS. ${ }^{2}$ The PBE density functional is used along with the aug-cc-pVDZ basis set in all calculations except noted in the text.

Table S1. Details of the grid levels used in the main text. Grid selection was made on the basis of atom types, in which $\mathrm{C}, \mathrm{O}$ and $\mathrm{N}$ are classed in one group. The first number in brackets corresponds to the number of radial points and the second number refers to Lebedev order.

\begin{tabular}{ccc}
\hline & $\mathrm{H}$ & $\mathrm{C} / \mathrm{O} / \mathrm{N}$ \\
\hline Level 1 & $(30,17)$ & $(40,23)$ \\
Level 2 & $(40,23)$ & $(60,29)$ \\
Level 4 & $(60,35)$ & $(90,41)$ \\
\hline
\end{tabular}

2. Assessment of the Yukawa potential and erf function for Coulomb damping. 
The Yukawa potential and error function are both widely used for Coulomb screening in range-separation DFT methods. In fact, the erf-function was considered in our work, with which the scaling factor $F[\rho(r)]$ has the following form

$$
F\left[a_{\sigma}\right]=1-\frac{8}{3} a_{\sigma}\left[\sqrt{\pi} \operatorname{erf}\left(\frac{1}{2 a_{\sigma}}\right)+\left(2 a_{\sigma}-4 a_{\sigma}^{3}\right)-\exp \left(-\frac{1}{4 a_{\sigma}^{2}}\right)-4 a+4 a_{\sigma}^{3}\right] \text {. }
$$

where $a_{\sigma}=\mu / 2(6 \pi \rho)^{1 / 3}$ with $\underline{\sigma}$ denoting the spin coordinate, and $\mu$ is the screening factor. We have also obtained an optimal value of $\mu$, which is 0.258 . Shows in Figure $\mathrm{S} 1$ is a comparison of the performance of the Yukawa potential and the error function, and we do not find statistically significant difference between the two. The expression of the Yukawa potential is explicit, easy for future modifications. So, we decided to use the Yukawa potential in XFP.

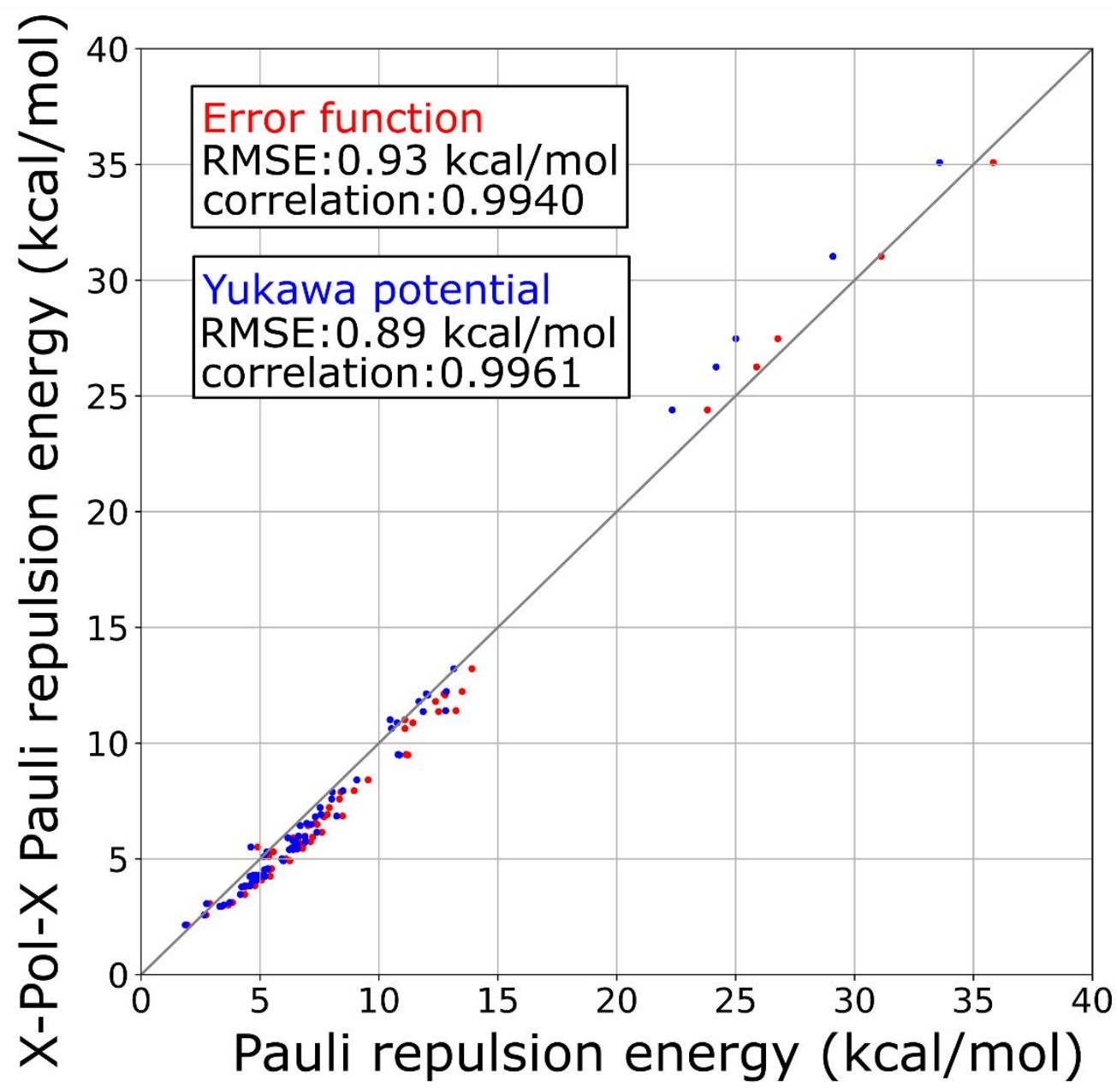


Figure S1. Pauli repulsion energy from XFP compared with the exact X-Pol-X Pauli repulsion energy. Red dots denote results computed using the erf screening scheme, and blue points refer to results from the Yukawa potential scheme.

Range-separation in KS-DFT with Gaussian-type basis functions (GTF) does indeed prefer using the error function, because the long-range electron exchange integrals (4-centers) with the error function over GTF can be computed more easily than those with the Yukawa potential. However, the 4-center, long-range exchange integrals are not required in case. In principle, both Yukawa potential and error function can be used in our XFP.

\section{Assignment of numerical grid levels.}

Numerical grids for computing the repulsion integrals of the present fragment exchange potential were generated using standard grid procedures in Kohn-Sham density functional theory (DFT) calculations. The M4 Mapping scheme described in ref. ${ }^{3}$ is applied in radial integration and the Lebedev grids are employed for spherical integration. Levels in the text (see Table 1) refers to the number of grid points that are deployed on radial and spherical integrations. Becke's scheme were used in grids partition. The details of levels are listed in table 1.

\section{Optimization of the screening parameter $(\gamma)$ in the Yukawa potential}

.First, a smaller dataset, the S66 database, is selected as training set, which contains 66 bimolecular geometries at the equilibrium (minimum) intermolecular distance. We adjusted the screening parameter $(\gamma)$ to minimize the difference between Pauli deformation described by fragmental exchange potential $\Delta \rho^{\mathrm{XFP}}(\mathbf{r})$, and that from 
orthogonalization $\Delta \rho^{\text {orth. }}$. The difference is measured by the root-mean-square-error (RMSE) of the electron density deformation between the target (orthogonalization) and the XFP potential, defined by

$$
\delta \rho_{m}=\int\left|\Delta \rho_{m}^{\text {orth. }}(\mathbf{r})-\Delta \rho_{m}^{\mathrm{XFP}}(\mathbf{r})\right| d \mathbf{r}
$$

where $\mathrm{m}$ is a bimolecular complex in the S66 database.

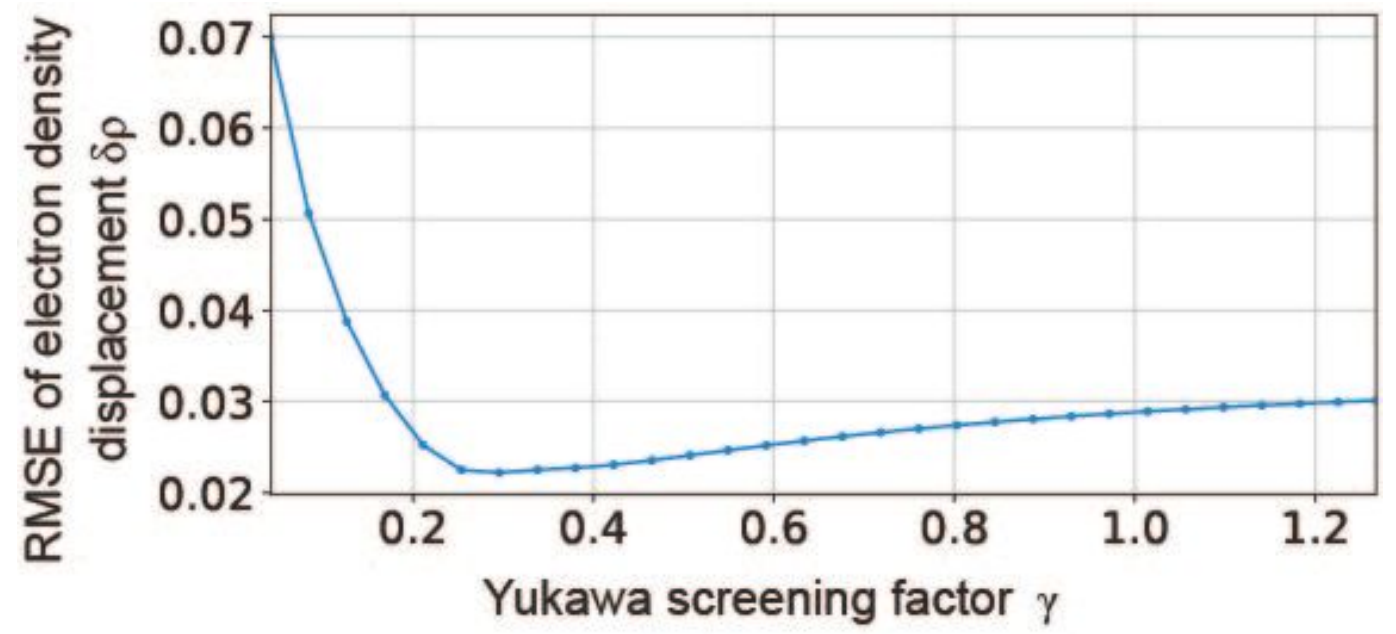

Figure S2. Root-mean-square-error between computed Pauli deformation density and that using the exact explicit polarization with exchange method for the S66 bimolecular complex set.

The screening parameters $(\gamma)$ are scanned from 0 to 1.4 , where 0 means that there is no Coulomb screening and that a large $\gamma$ refers to strong Coulomb screening. The RMSE of electron density misplacement $\delta \rho$ is plotted in figure S2. We found that the optimum $\gamma$ is located at the range of $0.2-0.3$. 

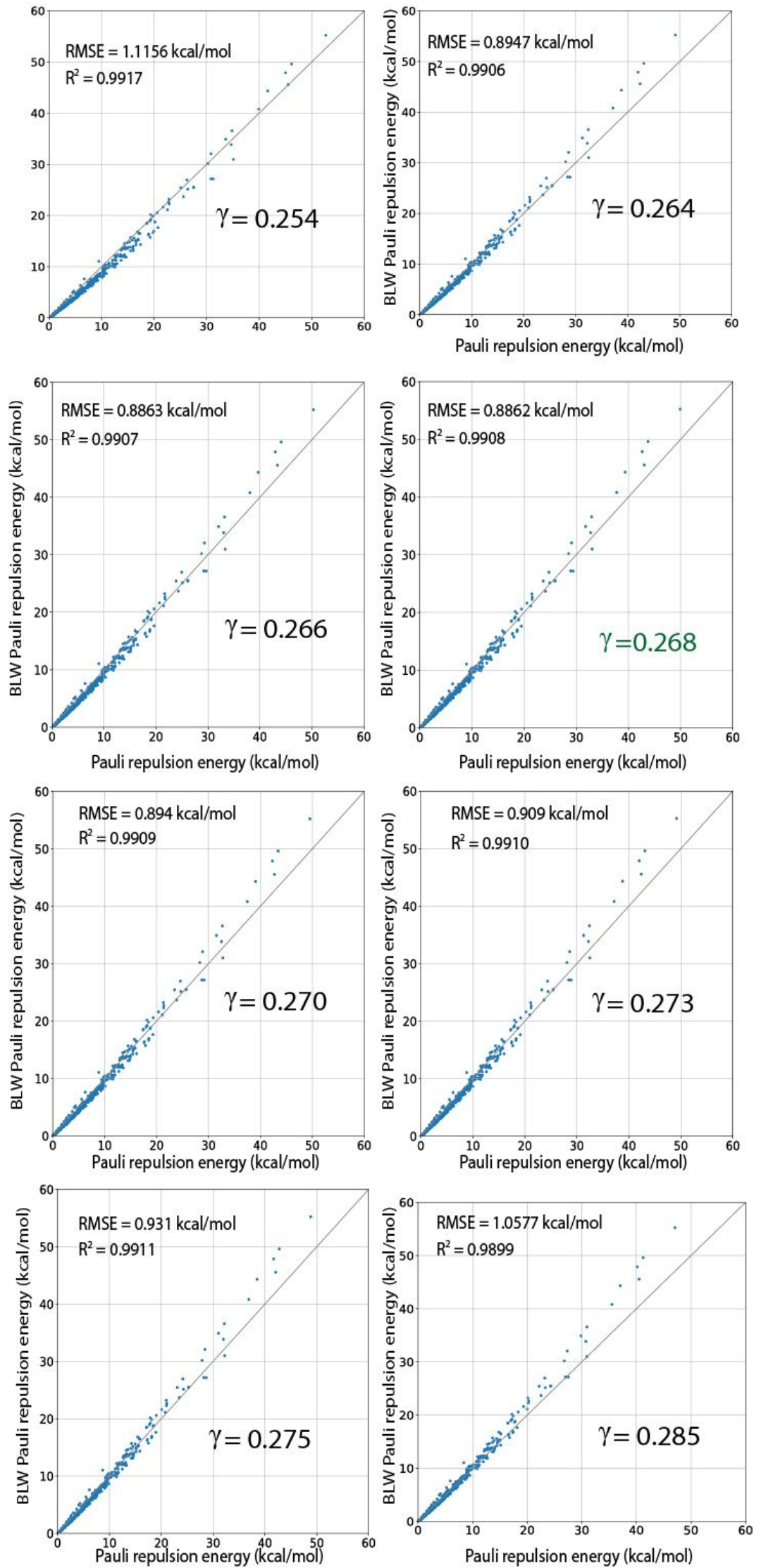

Figure S3. Comparison of the Pauli repulsion energy from the XFP potential using various $\gamma$ values and from the BLW-ED (X-Pol-X) exchange energy for the S66x8 dataset of 528 bimolecular complexes. 
After locating the optimum range of $\gamma$. We used a larger dataset, the S66 $\times 8$ database, which contains 528 bimolecular geometries both at the equilibrium (minimum) intermolecular distance and arbitrary separations, to further search for the specific value of $\gamma$. The optimization strategy was switched to minimizing the RMSE of exchange repulsion energy in comparison with results from the X-Pol-X method. The comparison between XFP and X-Pol-X (equivalent to the BLW-ED exchange term) under different $\gamma$ values is listed in figure S3. We found that $\gamma=0.268$ is the optimum value, which is used throughout.

1. (a) Sun, Q., Libcint: An efficient general integral library for Gaussian basis functions. J. Comput. Chem. 2015, 36 (22), 1664-1671; (b) Sun, Q.; Berkelbach, T. C.; Blunt, N. S.; Booth, G. H.; Guo, S.; Li, Z.; Liu, J.; McClain, J. D.; Sayfutyarova, E. R.; Sharma, S.; Wouters, S.; Chan, G. K.-L., PySCF: the Python-based simulations of chemistry framework. WIREs Computational Molecular Science 2018, 8 (1), e1340.

2. Schmidt, M. W.; Baldridge, K. K.; Boatz, J. A.; Elbert, S. T.; Gordon, M. S.; Jensen, J. H.; Koseki, S.; Matsunaga, N.; Nguyen, K. A.; Su, S. J.; Windus, T. L.; Dupuis, M.; Montgomery, J. S., General atomic and molecular electronic structure system. J. Comput. Chem. 1993, 14 (11), 1347-63.

3. Treutler, O.; Ahlrichs, R., Efficient molecular numerical integration schemes. The Journal of Chemical Physics 1995, 102 (1), 346-354.

4. Viquez Rojas, C. I.; Fine, J.; Slipchenko, L. V., Exchange-Repulsion Energy in Qm/Efp. J. Chem. Phys. 2018, 149, 094103.

\section{Structures of the dimers with large errors.}

Eight dimers with unsigned error larger than $3 \mathrm{kcal} / \mathrm{mol}$ are plotted below. The error is defined as,

$$
\text { Error }=E_{\mathrm{XFP}}^{r e p .}-E_{\mathrm{X}-\mathrm{Pol}-\mathrm{X}}^{r e p}
$$




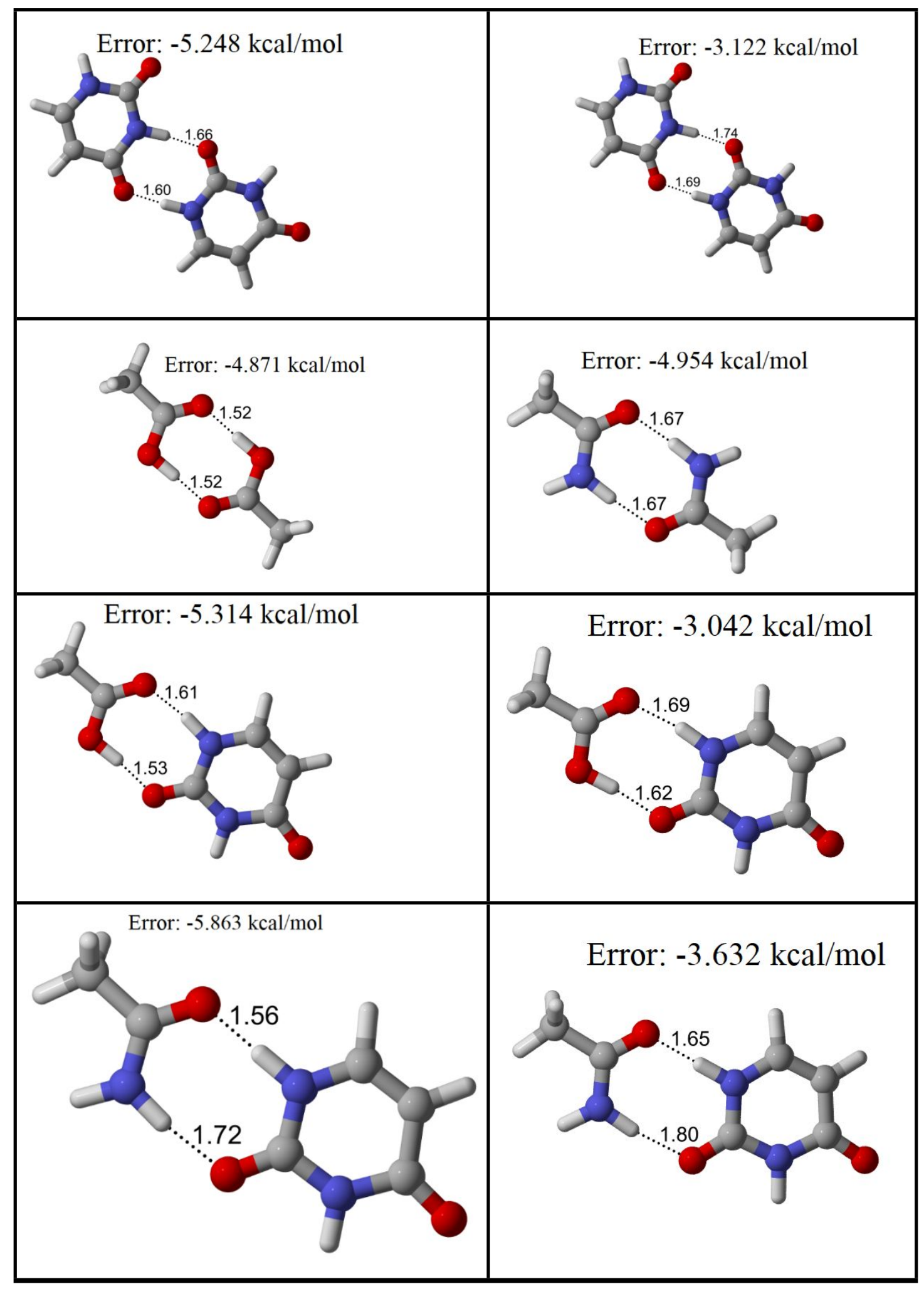




\section{Effect of exchange polarization on electron density and comparison with KS-DFT total density.}

To describe the total density in high accuracy is an ultimate target of fragmentbased method. The existence of Pauli deformation does improve the performance of fragment-based method. Taking the X-Pol method as an example, the difference between the densities described by X-Pol without intermolecular exchange and KSDFT calculation,

$$
\Delta \rho=\rho^{\mathrm{X}-\mathrm{Pol}}-\rho^{\mathrm{KS}-\mathrm{DFT}}
$$

is reduced by introducing XFP as in the following figure. Without exchange repulsion, the electrons are attracted to move towards positive charge region. 

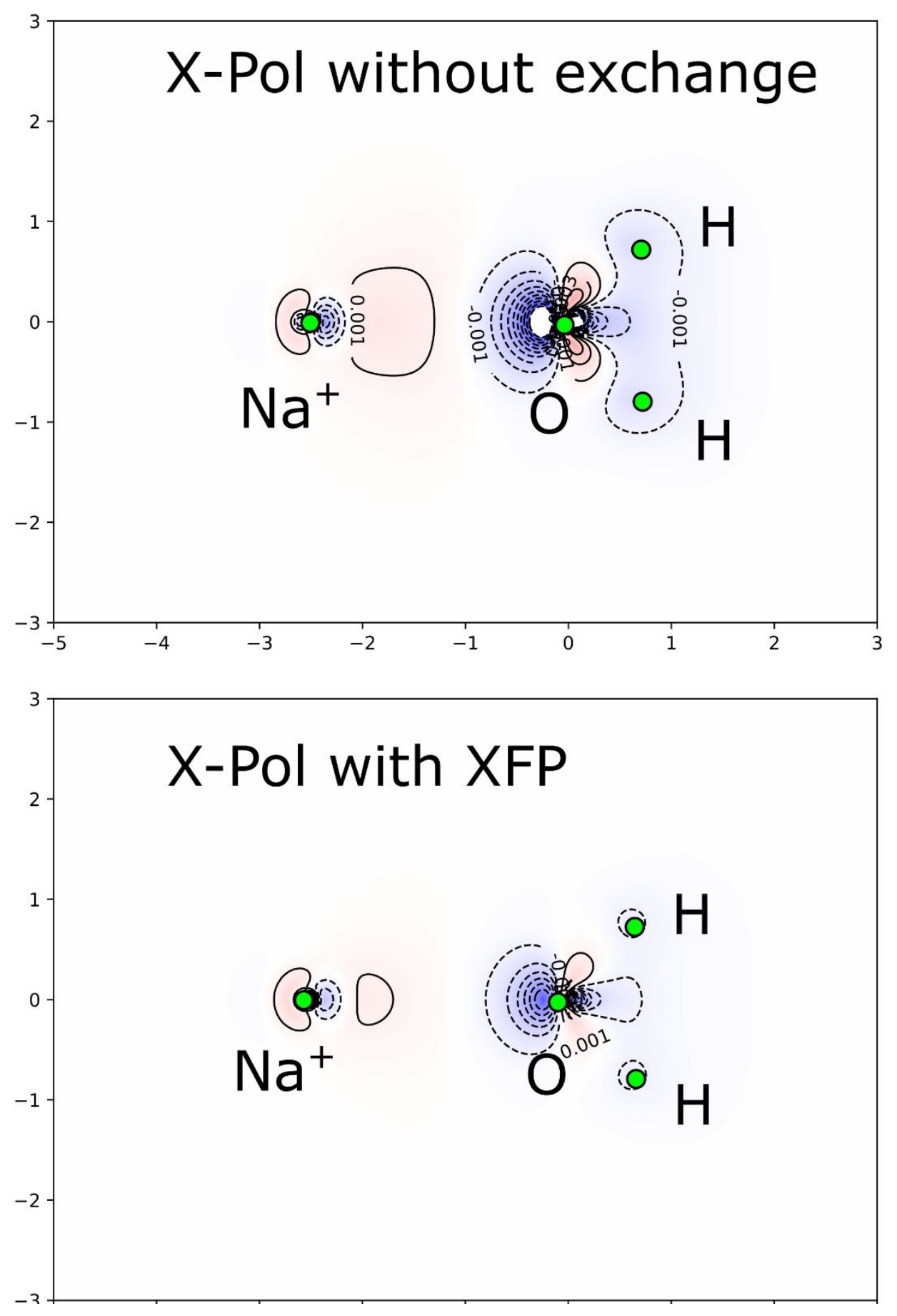

Figure 2. Density difference contours computed without (top) and with (bottom) exchange effects relative to that of KS-DFT using PBE/aug-cc-pVDZ. The $\mathrm{Na}^{+}-$ water complex at the optimal geometry is used for the illustration. 